\title{
Controversial Issues in Thyroid Cancer Management
}

\author{
R. Michael Tuttle \\ Endocrinology Service, Memorial Sloan Kettering Cancer Center, New York, New York
}

Learning Objectives: On successful completion of this activity, participants should be able to describe (1) the option of thyroid lobectomy as initial therapy for
thyroid cancer; (2) the use of preoperative neck imaging to optimize the completeness of the initial surgery; and (3) the selective use of RAI for remnant ablation, adjuvant treatment, or treatment of known persistent or recurrent disease.

Financial Disclosure: This research was funded in part by NIH/NCI Cancer Center support grant P30 CA008748 (Craig Thompson, principal investigator) and Specialized Programs of Research Excellence (SPORE) in Thyroid Cancer grant P50 CA172012-01A1 (James Fagin, principal investigator). The author of this article has indicated no other relevant relationships that could be perceived as a real or apparent conflict of interest.

CME Credit: SNMMI is accredited by the Accreditation Council for Continuing Medical Education (ACCME) to sponsor continuing education for physicians. SNMMI designates each JNM continuing education article for a maximum of 2.0 AMA PRA Category 1 Credits. Physicians should claim only credit commensurate with the extent of their participation in the activity. For CE credit, SAM, and other credit types, participants can access this activity through the SNMMI website (http://www.snmmilearningcenter.org) through August 2021.

\section{See an invited perspective on this article on page 1184}

The lack of prospective randomized clinical trials for most management topics in differentiated thyroid cancer forces us to make management recommendations based on retrospective observational data, which are often incomplete, subject to selection bias, and conflicting. Therefore, it is not surprising that many aspects of thyroid cancer management remain controversial and not well defined. This review will examine the controversies surrounding 3 important topics in thyroid cancer management: the option of thyroid lobectomy as initial therapy, the use of preoperative neck imaging to optimize the completeness of the initial surgery, and the selective use of radioactive iodine for remnant ablation, adjuvant treatment, or treatment for known persistent or recurrent disease. As thyroid cancer management moves toward a much more risk-adapted approach to personalized recommendations, clinicians and patients must balance the risks and benefits of the potential options to arrive at a plan that is optimized regarding both patient preferences/values and the philosophy/experience of the local disease management team.

Key Words: thyroid cancer; radioactive iodine therapy; thyroid lobectomy

J Nucl Med 2018; 59:1187-1194

DOI: 10.2967/jnumed.117.192559

$\mathbf{T}$

hyroid cancer management has seen a dramatic shift away from a one-size-fits-all approach to a more risk-adapted treatment paradigm in which the extent of surgery, the use of radioactive iodine (RAI) therapy, the goals for thyroid-stimulating hormone suppression, and follow-up recommendations are individually tailored for each patient (1-7). In the past, essentially all patients except those with intrathyroidal papillary microcarcinoma were

Received Feb. 6, 2018; revision accepted Apr. 9, 2018.

For correspondence or reprints contact: Michael Tuttle, Endocrinology Service, Department of Medicine, Memorial Sloan Kettering Cancer Center, 1275 York Ave., New York, NY 10021.

E-mail: tuttlem@mskcc.org

Published online Apr. 13, 2018.

COPYRIGHT (C) 2018 by the Society of Nuclear Medicine and Molecular Imaging. subjected to high-intensity treatment (total thyroidectomy with or without prophylactic central neck dissection, RAI therapy, prolonged thyroid-stimulating hormone suppression, and highly sensitive followup evaluations). More recently, the thyroid cancer guidelines of both the American Thyroid Association (ATA) and the National Comprehensive Cancer Network have evolved to allow for low-intensity treatment options (observation or thyroid lobectomy without the need for RAI therapy or thyroid-stimulating hormone suppressive therapy) in properly selected patients $(1,5)$. Even though these recommendations are often based on limited or conflicting data, the increasing incidence of low-risk thyroid cancers, which are commonly associated with an indolent disease course; the increasing reliance on neck ultrasonography rather than routine follow-up diagnostic whole-body RAI scanning for disease detection; and a more selective use of postoperative RAI has forced clinicians to seriously question whether intensive treatment and follow-up are mandatory for all differentiated thyroid tumors larger than $1 \mathrm{~cm}$.

Many clinicians and patients strongly favor high-intensity treatment options under the assumption that up-front aggressive treatments and early detection of small-volume residual disease would lead to improved outcomes. Conversely, advocates of low-intensity treatment options are confident that with proper patient selection and follow-up, similar long-term outcomes can be achieved. The effectiveness of the low-intensity treatment option is rooted in the following general assumptions: that most patients with low-risk differentiated thyroid cancer will do exceptionally well, with disease-specific survival rates in excess of $99 \%$ and structural disease recurrence rates of less than $5 \%-10 \%$ after either low-intensity or high-intensity treatments; that delayed intervention (surgery, RAI therapy), when necessary, is effective and has no impact on disease-specific survival; that minimal residual disease is common and of little clinical importance; and that early detection and treatment of very small volume persistent or recurrent structural disease has little clinical benefit.

Unfortunately, the literature comparing low-intensity with highintensity treatments is largely retrospective, observational, and devoid of high-quality prospective randomized clinical trials. However, it is interesting that despite more than 3 decades of increasingly sensitive disease-detection tools (such as highresolution neck ultrasonography and ultrasensitive thyroglobulin assays), which led to even more aggressive therapeutic interventions (such as prophylactic and therapeutic neck dissections for 
small-volume disease and repeated administrations of RAI for biochemical evidence of persistent disease), thyroid cancer mortality remains stable or slightly increased (8). Thus, the controversy will continue as thyroid cancer experts review the same body of literature and reach differing conclusions based on their training, clinical perspective, experience, and personal bias.

This review will examine the controversies surrounding 3 important topics in thyroid cancer management: the option of thyroid lobectomy as initial therapy, the use of preoperative neck imaging to optimize the completeness of the initial surgical procedure, and the selective use of RAI for remnant ablation, adjuvant treatment, or treatment for known persistent or recurrent disease.

\section{WHEN IS LOBECTOMY A REASONABLE INITIAL STRATEGY IN DIFFERENTIATED THYROID CANCER?}

The controversy: whether total thyroidectomy should be recommended as the primary surgical procedure in all differentiated thyroid cancers with primary tumors larger than $1 \mathrm{~cm}$ in maximal dimension.

\section{Position 1}

Total thyroidectomy should be recommended for all differentiated thyroid cancers with primary tumors larger than $1 \mathrm{~cm}$ in maximal dimension because complete removal of the thyroid gland may improve survival, decrease recurrence, allow for routine use of RAI therapy, and facilitate detection of recurrent or persistent disease during follow-up. The increased risk of surgical complications associated with total thyroidectomy can be minimized if the procedure is done by experienced surgeons.

\section{Position 2}

Total thyroidectomy is not required for differentiated thyroid cancers with primary tumors smaller than $4 \mathrm{~cm}$ in maximal dimension that appear to be confined to the thyroid gland on preoperative evaluation, because lobectomy is associated with the same excellent overall survival as complete removal of the gland, has a lower risk of surgical complications, and may avoid the need for full-replacement-dose thyroid hormone therapy in properly selected patients. Although the recurrence rate may be slightly higher after lobectomy, follow-up procedures can easily identify the few patients who demonstrate structural disease recurrence, making subsequent salvage therapy effective.

The primary point of controversy with regard to initial surgical management revolves around recommendation 35B (Table 1) in the ATA guidelines (1), allowing for lobectomy in patients with tumors between 1 and $4 \mathrm{~cm}$ without evidence of extrathyroidal extension or metastatic disease. Although the National Comprehensive Cancer Network guidelines have long allowed for lobectomy in this setting, the ATA guidelines had previously strongly recommended total thyroidectomy for tumors larger than $1 \mathrm{~cm}$ $(5,9,10)$. This recommendation was largely based on the assumption that nearly all of these patients would require postoperative RAI therapy and was further strengthened by the 2007 paper of Bilimoria et al. that demonstrated a statistically significant improvement in overall survival within this size range (10 y survival of $98.4 \%$ for total thyroidectomy vs. $97.1 \%$ for lobectomy, $P<0.05$ ) (11). Since that publication, multiple other studies have demonstrated no statistically significant survival benefit for total thyroidectomy versus lobectomy in tumors smaller than $4 \mathrm{~cm}$ when patients are properly selected and the statistical analysis controls for important confounding variables (Table 2). The specifics of the various retrospective studies examining this issue are extensively reviewed in the text of the ATA guidelines (1).

Even though total thyroidectomy may not be associated with a proven overall survival benefit in properly selected patients with 1to 4-cm intrathyroidal primary tumors, there are still valid reasons to consider a bilateral surgical procedure for individual patients. As noted in recommendation 35B (Table 1), total thyroidectomy would be the preferred operation if the disease management team thinks there will be a need for postoperative RAI therapy based on either disease features or patient preferences. This recommendation provides the flexibility to actively incorporate the thought processes of the local disease management team and the preferences of the patient into the decision about whether a lobectomy would be the most appropriate surgery for that individual patient.

TABLE 1

Operative Approach to Biopsy Diagnostic of Follicular Cell-Derived Malignancy (1)

Recommendation

no.

ATA guidance

35A For patients with thyroid cancer greater than $4 \mathrm{~cm}$, or with gross extrathyroidal extension (clinical T4), or clinically apparent metastatic disease to nodes (clinical N1) or distant sites (clinical M1), the initial surgical procedure should include a near-total or total thyroidectomy and gross removal of all primary tumor unless there are contraindications to this procedure. (Strong recommendation, Moderate quality evidence)

35B For patients with thyroid cancer $>1 \mathrm{~cm}$ and $<4 \mathrm{~cm}$ without extrathyroidal extension, and without clinical evidence of any lymph node metastases (cN0), the initial surgical procedure can be either a bilateral procedure (near-total or total thyroidectomy) or a unilateral procedure (lobectomy). Thyroid lobectomy alone may be sufficient initial treatment for low risk papillary and follicular carcinomas; however, the treatment team may choose total thyroidectomy to enable radioactive iodine therapy or to enhance follow-up based on disease features and/or patient preferences. (Strong recommendation, Moderate quality evidence)

$35 \mathrm{C}$

If surgery is chosen for patients with thyroid cancer $<1 \mathrm{~cm}$ without extrathyroidal extension and cNO, the initial surgical procedure should be a thyroid lobectomy unless there are clear indications to remove the contralateral lobe. Thyroid lobectomy alone is sufficient treatment for small, unifocal, intrathyroidal carcinomas in the absence of prior head and neck irradiation, familial thyroid carcinoma, or clinically detectable cervical nodal metastases. (Strong recommendation, Moderate quality evidence) 
TABLE 2

Survival Benefit for Total Thyroidectomy $(<4 \mathrm{~cm})$ Over Thyroid Lobectomy in Differentiated Thyroid Cancer

\begin{tabular}{lrll}
\hline $\begin{array}{c}\text { Retrospective } \\
\text { study }\end{array}$ & \multicolumn{1}{c}{$n$} & \multicolumn{1}{c}{ Source } & $\begin{array}{c}\text { Survival } \\
\text { benefit }\end{array}$ \\
\hline Bilimoria et al. (11) & 52,173 & NCDB & Yes \\
\hline Adam et al. (31) & 61,775 & NCDB & No \\
\hline Haigh et al. (32) & 5,432 & SEER & No \\
\hline Barney et al. (33) & 23,605 & SEER & No \\
$\begin{array}{l}\text { Mendelsohn et al. } \\
\text { (34) }\end{array}$ & 22,724 & SEER & No \\
\hline Nixon et al. (12) & 889 & Single center & No \\
\hline Matsuzu et al. (35) & 1,088 & Single center & No \\
\hline
\end{tabular}

NCDB = National Cancer Database; SEER = Surveillance, Epidemiology, and End Results Program.

Furthermore, since papillary thyroid carcinoma is known to be a multifocal disease, it is not surprising that total thyroidectomy is associated with a slightly lower risk of recurrence than lobectomy. However, with proper patient selection, locoregional recurrence rates of less than $1 \%-4 \%$ can be achieved in experienced centers that couple high-quality preoperative imaging (primarily neck ultrasonography) with sound clinical judgment $(7,12-14)$. Furthermore, salvage therapy is effective in the few low-risk patients who experience structural disease recurrence after low-intensity therapy, resulting in the same excellent survival rates that would have been expected from up-front high-intensity therapy.

It is also important for patients to understand that even if the preoperative plan is to proceed with a lobectomy, there are intraoperative and postoperative findings that may require conversion to a total thyroidectomy. Patients are encouraged to empower the surgeon to convert the surgery from a lobectomy to a total thyroidectomy on the basis of intraoperative findings. Likewise, it is important to emphasize that a final decision on the appropriateness of lobectomy as the initial surgical procedure can be determined only once the final histopathology report has been received, a week or two after surgery. Early-completion thyroidectomy after lobectomy is required in only $5 \%-6 \%$ of our patients $(7,12-14)$. However, disease management teams that are more aggressive in the use of RAI for intermediate-risk features may require earlycompletion thyroidectomy up to $20 \%$ of the time (15-17).

Using a clinical decision-making framework based on what we have previously published for active surveillance of very low risk thyroid cancers, we examine 3 critical interrelated domains to determine whether the patient would be a candidate for lobectomy (18): preoperative imaging and clinical findings, medical team characteristics, and patient characteristics.

Preoperative Imaging and Clinical Findings. The preoperative neck ultrasonography and the patient's history and physical examination are carefully reviewed. Any indication of gross extrathyroidal extension, locoregional metastasis, or distant metastasis leads to a recommendation for total thyroidectomy. Furthermore, the presence of multiple nodules in the contralateral lobe or extensive nonspecific findings on ultrasonography (such as chronic lymphocytic thyroiditis or atypical cervical lymph nodes) would increase the likelihood that a total thyroidectomy would be recommended to make follow-up evaluations easier.
The success of lobectomy as the initial treatment option is also significantly enhanced when experienced multidisciplinary management teams integrate sound clinical judgment with high-quality preoperative neck ultrasonography in the decision-making process. In the absence of high-quality neck ultrasonography, particularly with regard to the evaluation of abnormal cervical lymph nodes, the selection of patients for lobectomy will be suboptimal.

Medical Team Characteristics. It is important to understand the philosophy of the local disease management team with regard to the use of RAI in patients who demonstrate intermediate-risk histologic features that were not apparent on preoperative imaging (e.g., minor extrathyroidal extension, very small volume lymph node metastasis, and vascular invasion). Disease management teams that use RAI aggressively for intermediate risk factors will be expected to have a higher immediate-completion thyroidectomy rate than centers that use RAI much more selectively. This philosophy does not preclude the use of lobectomy as initial therapy, but the patient needs to understand what the likelihood is that unexpected histologic findings could lead to an earlycompletion thyroidectomy to allow for postoperative RAI therapy.

Patient Characteristics. In many practices, patients are actively involved in shared decision making with the disease management team to explore the risks and benefits of both surgical options. Some patients, referred to as medical maximalists (19), strongly prefer a total thyroidectomy in an effort to minimize the recurrence rate and maximize the sensitivity of disease detection during follow-up (these patients also often opt for routine use of RAI after surgery). Conversely, medical minimalists often choose lobectomy. They value the function of the contralateral lobe in order to avoid thyroid hormone replacement if at all possible. They are willing to accept the possibility that additional future treatments may be necessary but will opt for the least aggressive initial option that is associated with excellent outcomes. Likewise, they are minimalist in their approach to identifying and treating small-volume disease in the future and therefore do not require initial therapies designed simply to facilitate high-sensitivity follow-up. Thus, medical minimalists are often good candidates for low-intensity treatment options.

In summary, in the absence of a proven survival benefit to the routine use of total thyroidectomy in well-differentiated thyroid cancers in the 1- to 4-cm range, lobectomy continues to be a viable treatment option for properly selected patients. Proper patient selection requires high-quality preoperative evaluations coupled with a thorough understanding of both the medical team's characteristics and the patient's preferences. By integrating the multiple important factors encompassed in each of these 3 domains, the disease management team can arrive at the most appropriate initial management and follow-up strategy for an individual patient.

\section{WHEN IS IMAGING RECOMMENDED BEFORE INITIAL THYROID SURGERY?}

The controversy: whether CT with iodinated contrast agents should always be avoided before thyroid surgery.

\section{Position 1}

CT with iodinated contrast should always be avoided before thyroid surgery to avoid a delay in postoperative RAI administration.

\section{Position 2}

CT with iodinated contrast should routinely be performed in patients with locally aggressive disease or clinically apparent 
cervical lymph node metastases to optimize preoperative planning and the completeness of surgery. Imaging studies that optimize initial surgery are more important than early administration of RAI postoperatively.

One of the primary goals of initial therapy is to "remove the primary tumor, disease that has extended beyond the thyroid capsule, and clinically significant lymph node metastases" (1). Thus, preoperative evaluations must include anatomic assessments of the thyroid and neck to appropriately plan surgical interventions.

Neck ultrasonography is now widely used as the primary tool not only for evaluating thyroid nodules but also for examining cervical lymph nodes. The guidelines of both the National Comprehensive Cancer Network and the ATA recommend preoperative ultrasound to evaluate cervical lymph nodes in all patients undergoing thyroidectomy for confirmed or suspected malignancy (Table 3). Although extensive bilateral neck dissections can identify subclinical microscopic cervical lymph node metastasis in as many as $70 \%-80 \%$ of patients $(20)$, preoperative neck ultrasonography identifies suspicious cervical lymphadenopathy in about $20 \%-30 \%$ of cases $(21-26)$. As a result, routine use of preoperative ultrasound potentially alters the surgical management approach in at least $20 \%$ of the cases $(21,23,27)$. Routine preoperative ultrasonographic neck evaluations allow for a more complete initial surgical dissection that is expected to decrease subsequent recurrence rates and to facilitate the effectiveness of RAI therapy.

In addition to the routine use of neck ultrasonography in the preoperative evaluation of thyroid cancer, there are circumstances in which additional cross-sectional imaging with CT or MR scans is likely to yield important additional anatomic information (1). Clinical findings that would warrant cross-sectional imaging beyond ultrasonography include hoarseness, progressive dysphagia, a mass fixed to surrounding structures on examination, hemoptysis, stridor, rapid progression or enlargement of the tumor, sonographic suspicion of gross extrathyroidal extension, or bulky cervical lymphadenopathy. In these cases, a CT scan of the neck and chest with intravenous contrast is routinely recommended to carefully evaluate the lymph node basins because they extend from high in the neck down through the supraclavicular areas and into the upper mediastinum.

Unlike previous ATA-guideline additions that recommended avoiding iodinated intravenous contrast in the preoperative setting, recommendation 33A (Table 3 ) in the current guidelines clearly endorses the use of contrast when these high-risk situations are identified or suspected. Although there is no question that the use of iodinated contrast may delay the administration of postoperative RAI, it is thought that the "benefit gained from improved anatomic imaging generally outweighs any potential risk of a several week delay in RAI imaging or therapy" (1). Although the ATA guidelines correctly pointed out that urinary iodine levels usually return to normal within 4-8 wk after intravenous contrast administration (28), the precise period necessary to ensure that the previous contrast will have no effect on subsequent RAI therapy has not been precisely defined. There remains some concern that even though the urinary iodine has returned to normal, residual iodine in thyroid tissues could decrease the effectiveness of RAI therapy. Nonetheless, since a complete and comprehensive surgical removal of the thyroid and local regional metastasis is such an important component to the initial therapy, the use of intravenous contrast to optimize surgical planning is justified in patients who present with highrisk disease. A delay in the administration of therapeutic RAI for several months postoperatively is acceptable in order to achieve this potential benefit. Delaying RAI for a few months after intravenous contrast has not been associated with impairment in the effectiveness of RAI when used for ablation, adjuvant therapy, or treatment of known disease.

Additional preoperative imaging is seldom indicated in patients with differentiated thyroid carcinoma, unless they present with clinical signs or symptoms of distant metastasis. Although ${ }^{18} \mathrm{~F}-$ FDG PET scanning is a valuable tool for both initial staging and follow-up, it is largely restricted to RAI-refractory disease and seldom plays a role in preoperative staging for differentiated thyroid cancer $(1)$.

TABLE 3

Role of Preoperative Staging with Diagnostic Imaging and Laboratory Tests (1)

Recommendation

no.

\author{
ATA guidance
}

\begin{tabular}{ll}
\hline 32A & $\begin{array}{l}\text { Preoperative neck ultrasound for cervical (central and especially lateral neck compartments) lymph nodes is } \\
\text { recommended for all patients undergoing thyroidectomy for malignant or suspicious for malignancy cytologic } \\
\text { or molecular findings. (Strong recommendation, Moderate quality evidence) }\end{array}$ \\
32B & $\begin{array}{l}\text { Ultrasound guided fine-needle aspiration of sonographically suspicious lymph nodes } \geq 8-10 \text { mm in the smallest } \\
\text { diameter should be performed to confirm malignancy if this would change management. (Strong } \\
\text { recommendation, Moderate quality evidence) }\end{array}$ \\
32C & $\begin{array}{l}\text { The addition of fine-needle aspiration thyroglobulin washout in the evaluation of suspicious cervical lymph nodes } \\
\text { is appropriate in selected patients, but interpretation may be difficult in patients with an intact thyroid gland. } \\
\text { (Week recommendation, Low quality evidence) }\end{array}$ \\
33A & $\begin{array}{l}\text { Preoperative use of cross-sectional imaging studies (CT, MRI) with intravenous contrast is recommended as an } \\
\text { adjunct to ultrasound for patients with clinical suspicion for advanced disease, including invasive primary tumor, } \\
\text { or clinically apparent multiple or bulky lymph node involvement. (Strong recommendation, Low quality evidence) }\end{array}$ \\
33B & $\begin{array}{l}\text { Routine preoperative 18 FDG-PET scanning is not recommended. (Strong recommendation, Low quality } \\
\text { evidence) }\end{array}$ \\
\hline
\end{tabular}


TABLE 4

Goals of RAI Therapy in Differentiated Thyroid Cancer (1)

\begin{tabular}{|c|c|c|}
\hline RAI therapy type & Goal & Potential benefit \\
\hline Remnant ablation & $\begin{array}{l}\text { Destroy normal thyroid tissue remaining } \\
\text { after total thyroidectomy }\end{array}$ & $\begin{array}{l}\text { Improve initial staging; facilitate } \\
\text { highly sensitive follow-up }\end{array}$ \\
\hline Adjuvant treatment & $\begin{array}{l}\text { Destroy subclinical microscopic tumor } \\
\text { deposits that may be present }\end{array}$ & $\begin{array}{l}\text { Decrease recurrence; improve } \\
\text { disease-specific survival }\end{array}$ \\
\hline \multirow[t]{3}{*}{$\begin{array}{l}\text { Treatment of known residual/ } \\
\text { recurrent disease }\end{array}$} & $\begin{array}{l}\text { Destroy known residual/recurrent } \\
\text { disease }\end{array}$ & $\begin{array}{l}\text { Achieve excellent response } \\
\text { (remission) }\end{array}$ \\
\hline & & Improve disease-specific survival \\
\hline & & Improve progression-free survival \\
\hline
\end{tabular}

\section{WHAT IS THE ROLE FOR POSTOPERATIVE RAI THERAPY?}

The controversy: whether postoperative RAI therapy should be routinely recommended after total thyroidectomy for patients with differentiated thyroid cancer.

\section{Position 1}

RAI therapy should be routinely recommended for all patients with differentiated thyroid cancer (except patients with intrathyroidal papillary microcarcinoma) to improve disease-specific survival, decrease disease recurrence, facilitate initial staging, and allow for highly sensitive follow-up testing.

\section{Position 2}

The use of RAI therapy in differentiated thyroid cancer should be selective and based on individual assessment of risk of disease recurrence, risk of disease-specific mortality, postoperative disease status, the likelihood of a significant impact on clinical outcome, the need for additional postoperative staging, the need for highly sensitive follow-up evaluations, the side effect profile, the treatment philosophy of the local disease management team, and consideration of the patient's preferences and values.

Recommendations on the indications for postoperative RAI therapy continue to be one of the most controversial of issues in the management of differentiated thyroid cancer. To help frame a rational discussion, the goal of RAI therapy should be described as either remnant ablation, adjuvant treatment, or treatment of known residual or recurrent disease (Table 4) (29). With this nomenclature, $R A I$ therapy is used as a general term that encompasses the specific goals of remnant ablation, adjuvant treatment, and treatment of known residual or recurrent disease. It is important to remember that since adjuvant treatment is given for a risk, rather than for demonstrable disease, it is accepted that a proportion of patients who receive adjuvant treatment will already have been cured by their primary surgery. Therefore, selection for adjuvant treatment involves both an assessment of the risk of recurrence or disease-specific mortality and a prediction of the likelihood that the proposed treatment will have a meaningful impact on these event rates.

Several aspects of the current literature make it exceedingly difficult to confidently determine whether RAI therapy will have a significant impact on recurrence or disease-specific survival (Fig. 1) (30). As a result, most guidelines provide general advice as to which intermediate-risk patients may benefit from RAI while giving definitive recommendations for only those patients with very low or high risk disease.
Before making specific recommendations on the potential benefit of RAI therapy in the postoperative setting, the ATA guidelines specifically recommend a careful evaluation of the postoperative disease status to determine whether additional therapies or imaging may be appropriate (Table 5) (1). This evaluation will provide information to determine whether the goal of RAI therapy would be ablation, adjuvant treatment, or treatment of known residual or recurrent disease and thereby the specific activity that would be administered.

Even though the ATA guidelines fully endorse the importance of postoperative disease status as a key factor in the decisionmaking process, the recommendations with regard to when clinicians should consider ablation or adjuvant therapy are largely based on ATA and TNM risk stratification (Table 6) (1). However, the ATA guidelines also recognize that "[i]n addition to standard clinicopathologic features, local factors such as the quality of preoperative and postoperative [ultrasound] evaluations, availability and quality of [thyroglobulin] measurements, experience of the operating surgeon, and clinical concerns of the local disease management team may also be considerations in postoperative RAI decision-making" (1). In addition, recommendations 51A and 51C (Table 6) also emphasize that patient preferences are relevant to RAI decision making.

From a practical standpoint, assessment of at least 9 key factors is required to determine whether postoperative RAI therapy is

\footnotetext{
- There is a lack of randomized controlled trials

- More than 16 different staging systems are used to define "risk"

- Definition of "low risk" varies across studies

- Few studies include assessment of postoperative disease status

- Few data are specific to the "adjuvant treatment" cohort

- Histologic heterogeneity exists in differentiated thyroid cancer

- Likelihood of RAI avidity is not considered

- Event rates are often small

- Events can occur decades after diagnosis

- Preoperative imaging has improved over time

- Methods for detecting recurrent disease have improved over time
}

FIGURE 1. Factors that make interpretation of the current literature difficult. Multiple factors make it difficult to use numerous retrospective, observational studies to arrive at definite conclusions on optimal use of RAl therapy. (Adapted from Sacks et al. (30).) 
TABLE 5

Role of Postoperative Disease Status in Decision Making About RAI Therapy for Differentiated Thyroid Cancer (1)

\title{
Recommendation
}

no.

ATA guidance

\begin{abstract}
50A Postoperative disease status (i.e., the presence or absence of persistent disease) should be considered in deciding whether additional treatment (e.g., RAl, surgery, or other treatment) may be needed. (Strong recommendation, Low quality evidence)

50B Postoperative serum thyroglobulin (on thyroid hormone therapy or after TSH stimulation) can help in assessing the persistence of disease or thyroid remnant and predicting potential future disease recurrence. The thyroglobulin should reach its nadir by 3 to 4 weeks postoperatively in most patients (Strong recommendation, Moderate quality evidence)
\end{abstract}

50C The optimal cutoff for postoperative serum thyroglobulin or state in which it is measured (on thyroid hormone therapy or after TSH stimulation) to guide decision-making regarding RAl administration is not known. (No recommendation, Insufficient evidence)

50D Postoperative diagnostic radioactive iodine whole body scans may be useful when the extent of the thyroid remnant or residual disease cannot be accurately ascertained from the surgical report or neck ultrasonography, and when the results may alter the decision to treat or the activity of radioactive iodine that is to be administered. Identification and localization of uptake foci may be enhanced by concomitant single photon emission computed tomography- computed tomography (SPECT/CT). When performed, pre-therapy diagnostic scan should utilize ${ }^{123} \mathrm{I}\left(1.5\right.$ to $3 \mathrm{mCi}$ ) or a low activity of ${ }^{131} \mathrm{I}$ ( 1 to $3 \mathrm{mCi}$ ) with the therapeutic activity optimally administered within 72 hours of the diagnostic activity. (Week recommendation, Low quality evidence)

$1 \mathrm{mCi}=37 \mathrm{MBq}$.

likely to benefit a specific patient: risk of disease recurrence; risk of disease-specific mortality; postoperative disease status (including the presence or absence of antithyroglobulin antibodies); the likelihood of a significant impact on the outcomes of interest (recurrence, disease-specific mortality); the likelihood of a significant improvement in initial staging; the need to facilitate highly sensitive follow-up evaluations; the potential side effect profile; patient preferences and values; and local factors such as the quality of ultrasound evaluations and thyroglobulin measurements, the experience of the operating team, and the treatment philosophy of the disease management team.

This management approach generally leads to a recommendation for observation in ATA low-risk patients who have no evidence of persistent disease after initial surgery and to a recommendation for RAI therapy in most ATA high-risk patients, either for adjuvant therapy or for treatment of known disease (Table 6) (1). RAI treatment should be "considered" in ATA intermediate-risk

TABLE 6

Role of RAI* after Thyroidectomy in Primary Management of Differentiated Thyroid Cancer (1)

Recommendation

no.
ATA guidance
$51 \mathrm{~A}$

Radioactive iodine remnant ablation is not routinely recommended after thyroidectomy for patients with multifocal papillary microcarcinoma in the absence of other adverse features. Consideration of specific features of the individual patient that could modulate recurrence risk, disease follow-up implications, and patient preferences are relevant to radioactive iodine decision-making. (Weak recommendation, Low quality evidence)

51D Radioactive iodine adjuvant therapy should be considered after total thyroidectomy in ATA intermediate risk level differentiated thyroid cancer patients. (Weak recommendation, Low quality evidence)

51E Radioactive iodine adjuvant therapy is routinely recommended after total thyroidectomy for ATA high risk differentiated thyroid cancer patients. (Strong recommendation, Moderate quality evidence)

*Including remnant ablation, adjuvant therapy, or therapy for persistent disease. 
TABLE 7

Recommended Administered Activity When RAI is Used for Remnant Ablation, Adjuvant Treatment, or Treatment of Known Residual Disease (1)

Recommendation

no.

ATA guidance

$55 \mathrm{~A}$

If radioactive iodine remnant ablation is performed after total thyroidectomy for ATA low risk thyroid cancer or intermediate risk disease with lower features (i.e., low-volume central neck nodal metastasis with no other known gross residual disease or any other adverse features), a low administered activity of approximately 30 $\mathrm{mCi}$ is generally favored over higher administered activities. (Weak recommendation, Low quality evidence)

55B Higher administered activities may need to be considered for patients receiving less than total or near total thyroidectomy in which a larger remnant is suspected or in which adjuvant therapy is intended. (Weak recommendation, Low quality evidence)

56 When radioactive iodine is intended for initial adjuvant therapy to treat suspected microscopic residual disease, administered activities above those used for remnant ablation up to $150 \mathrm{mCi}$ are generally recommended (in the absence of known distant metastases). It is uncertain whether routine use of higher administered activities (> $150 \mathrm{mCi}$ ) in this setting will reduce structural disease recurrence for T3 and N1 disease. (Weak recommendation, Low quality evidence)

77B The selection of RAl activity to administer for pulmonary micrometastasis can be empiric ( 100 to $200 \mathrm{mCi}$, or 100 to $150 \mathrm{mCi}$ for patients $\geq 70$ years old) or estimated by dosimetry to limit whole body retention to $80 \mathrm{mCi}$ at 48 hours and 200 cGy to the bone marrow. (Strong recommendation, Moderate quality evidence)

78 Radioiodine avid macronodular metastasis may be treated with radioactive iodine and treatment may be repeated when objective benefit is demonstrated (decrease in the size of the lesions, decreasing thyroglobulin), but complete remission is not common and survival remains poor. The selection of RAl activity to administer can be made empirically (100-200 $\mathrm{mCi}$ ) or by lesion dosimetry or whole body dosimetry if available to limit whole body retention to $80 \mathrm{mCi}$ at 48 hours and $200 \mathrm{cGy}$ to the bone marrow. (Weak recommendation, Low quality evidence)

79

With regard to treatment of bone metastases, the RAl activity administered can be given empirically (100 to 200 $\mathrm{mCi}$ ) or determined by dosimetry. (Weak recommendation, Low quality evidence)

$1 \mathrm{mCi}=37 \mathrm{MBq}$.

patients (recommendation 51D, Table 6). Whether RAI treatment is recommended in the ATA intermediate-risk patients will depend on their risk of recurrence and disease-specific mortality coupled with a careful evaluation of their postoperative disease status. Many ATA intermediate-risk patients will have persistent small-volume structural disease or abnormal postoperative thyroglobulin values that indicate persistent disease and thus would be candidates for treatment of known persistent disease with RAI. If the postoperative evaluation shows no evidence of biochemical or structural disease, then consideration is given as to whether remnant ablation or adjuvant therapy would be appropriate. The ATA intermediate-risk patients deemed to have a structural disease recurrence of about $5 \%$ or less would be considered for observation or possibly remnant ablation to facilitate initial staging and follow-up. Patients classified as having a higher risk of recurrence would be considered for adjuvant therapy in an effort to decrease the risk of recurrence and potentially improve overall survival based on whether the tumor is likely to be responsive to RAI. This logic integrates into the decision-making process the risk of recurrence, the risk of disease-specific mortality, the postoperative disease status, and the likelihood that adjuvant treatment would be effective.

Regarding administered activities, the ATA guidelines endorse approximately $1,110 \mathrm{MBq}$ of RAI as a reasonable choice for routine remnant ablation in low-risk patients (recommendation 55A, Table 7) (1). Higher administered activities are recommended when the goal is to achieve adjuvant therapy. Unfortunately, the currently available literature does not provide adequate guidance on the optimal administered activity for patients in the adjuvant therapy setting. Therefore, based primarily on expert opinion, the recommended activity for adjuvant therapy could vary anywhere from 1,110 to $5,550 \mathrm{MBq}$, depending on the multiple factors mentioned above and the philosophy of the local disease management team (recommendations 55B and 56, Table 7). For patients with known persistent or recurrent disease, administered activities in the 3,700- to 7,400-MBq range are generally recommended, except in the elderly, whose maximum administered activity should generally not exceed $5,550 \mathrm{MBq}(1)$.

\section{CONCLUSION}

Whereas many areas of controversy remain in the management of thyroid cancer, there are far more areas where general agreement has been achieved among all specialties. By carefully exploring the logic and rationale underlying the decision making that goes into management recommendations on the extent of initial surgery, the completeness of preoperative imaging, and the role of RAI therapy, clinicians can better understand the unique viewpoint that each specialty brings to this complex issue. Such an understanding will also allow us to further define specific areas needing additional research before definitive recommendations can be developed. It is only through proactive, purposeful, and 
inclusive interdisciplinary cooperation that this field can be moved forward as we strive to optimize the intensity of therapy and follow-up for each individual with thyroid cancer.

\section{REFERENCES}

1. Haugen BR, Alexander EK, Bible KC, et al. 2015 American Thyroid Association management guidelines for adult patients with thyroid nodules and differentiated thyroid cancer: The American Thyroid Association Guidelines Task Force on thyroid nodules and differentiated thyroid cancer. Thyroid. 2016;26: $1-133$.

2. Haymart MR, Esfandiari NH, Stang MT, Sosa JA. Controversies in the management of low-risk differentiated thyroid cancer. Endocr Rev. 2017;38:351378.

3. Momesso DP, Tuttle RM. Update on differentiated thyroid cancer staging. Endocrinol Metab Clin North Am. 2014;43:401-421.

4. Momesso DP, Vaisman F, Yang SP, et al. Dynamic risk stratification in patients with differentiated thyroid cancer treated without radioactive iodine. J Clin Endocrinol Metab. 2016;101:2692-2700.

5. NCCN clinical practice guidelines in oncology (NCCN Guidelines): thyroid carcinoma-version 2.2017. https://www.nccn.org/professionals/physician_gls/ pdf/thyroid.pdf. Accessed April 25, 2018.

6. Tuttle RM, Sabra MM. Selective use of RAI for ablation and adjuvant therapy after total thyroidectomy for differentiated thyroid cancer: a practical approach to clinical decision making. Oral Oncol. 2013;49:676-683.

7. Vaisman F, Momesso D, Bulzico DA, et al. Thyroid lobectomy is associated with excellent clinical outcomes in properly selected differentiated thyroid cancer patients with primary tumors greater than $1 \mathrm{~cm}$. J Thyroid Res. 2013; 2013:398194.

8. Lim H, Devesa SS, Sosa JA, Check D, Kitahara CM. Trends in thyroid cancer incidence and mortality in the United States, 1974-2013. JAMA. 2017;317:13381348.

9. Cooper DS, Doherty GM, Haugen BR, et al. Revised American Thyroid Association management guidelines for patients with thyroid nodules and differentiated thyroid cancer. Thyroid. 2009;19:1167-1214.

10. Cooper DS, Doherty GM, Haugen BR, et al. Management guidelines for patients with thyroid nodules and differentiated thyroid cancer. Thyroid. 2006;16:109142.

11. Bilimoria KY, Bentrem DJ, Ko CY, et al. Extent of surgery affects survival for papillary thyroid cancer. Ann Surg. 2007;246:375-381.

12. Nixon IJ, Ganly I, Patel SG, et al. Thyroid lobectomy for treatment of well differentiated intrathyroid malignancy. Surgery. 2012;151:571-579.

13. Nixon IJ, Palmer FL, Whitcher MM, et al. Thyroid isthmusectomy for welldifferentiated thyroid cancer. Ann Surg Oncol. 2011;18:767-770.

14. Vaisman F, Shaha A, Fish S, Tuttle R. Initial therapy with either thyroid lobectomy or total thyroidectomy without radioactive iodine remnant ablation is associated with very low rates of structural disease recurrence in properly selected patients with differentiated thyroid cancer. Clin Endocrinol (Oxf). 2011;75:112-119.

15. Calcatera NA, Lutfi W, Suman P, et al. Concordance of preoperative clinical stage with pathological stage in patients $\leq 45$ years with well-differentiated thyroid cancer. Endocr Pract. 2018;16:27-32.

16. Duh QY, Shen WT. Clinical implications of postoperative up-staging of differentiated thyroid cancer based upon pathologic evaluation. Endocr Pract. 2018; 16:124-125.
17. Kluijfhout WP, Pasternak JD, Drake FT, et al. Application of the new American Thyroid Association guidelines leads to a substantial rate of completion total thyroidectomy to enable adjuvant radioactive iodine. Surgery. 2017;161:127-133.

18. Brito JP, Ito Y, Miyauchi A, Tuttle RM. A clinical framework to facilitate risk stratification when considering an active surveillance alternative to immediate biopsy and surgery in papillary microcarcinoma. Thyroid. 2016;26:144-149.

19. Groopman J, Hartzband P. Your Medical Mind: How to Decide What Is Right for You. New York, NY: Penguin Books; 2011:211-218.

20. Wada N, Duh QY, Sugino K, et al. Lymph node metastasis from 259 papillary thyroid microcarcinomas: frequency, pattern of occurrence and recurrence, and optimal strategy for neck dissection. Ann Surg. 2003;237:399-407.

21. Kouvaraki MA, Shapiro SE, Fornage BD, et al. Role of preoperative ultrasonography in the surgical management of patients with thyroid cancer. Surgery. 2003; 134:946-954.

22. Leboulleux S, Girard E, Rose M, et al. Ultrasound criteria of malignancy for cervical lymph nodes in patients followed up for differentiated thyroid cancer. J Clin Endocrinol Metab. 2007;92:3590-3594.

23. O'Connell K, Yen TW, Quiroz F, Evans DB, Wang TS. The utility of routine preoperative cervical ultrasonography in patients undergoing thyroidectomy for differentiated thyroid cancer. Surgery. 2013;154:697-701.

24. Shimamoto K, Satake H, Sawaki A, Ishigaki T, Funahashi H, Imai T. Preoperative staging of thyroid papillary carcinoma with ultrasonography. Eur J Radiol. 1998;29:4-10

25. Solorzano CC, Carneiro DM, Ramirez M, Lee TM, Irvin GL III. Surgeonperformed ultrasound in the management of thyroid malignancy. Am Surg. 2004; 70:576-580.

26. Stulak JM, Grant CS, Farley DR, et al. Value of preoperative ultrasonography in the surgical management of initial and reoperative papillary thyroid cancer. Arch Surg. 2006;141:489-494.

27. Horvath E, Majlis S, Rossi R, et al. An ultrasonogram reporting system for thyroid nodules stratifying cancer risk for clinical management. J Clin Endocrinol Metab. 2009;94:1748-1751.

28. Padovani RP, Kasamatsu TS, Nakabashi CC, et al. One month is sufficient for urinary iodine to return to its baseline value after the use of water-soluble iodinated contrast agents in post-thyroidectomy patients requiring radioiodine therapy. Thyroid. 2012;22:926-930.

29. Van Nostrand D. Selected controversies of radioiodine imaging and therapy in differentiated thyroid cancer. Endocrinol Metab Clin North Am. 2017;46:783-793.

30. Sacks W, Fung CH, Chang JT, Waxman A, Braunstein GD. The effectiveness of radioactive iodine for treatment of low-risk thyroid cancer: a systematic analysis of the peer-reviewed literature from 1966 to April 2008. Thyroid. 2010;20:12351245 .

31. Adam MA, Pura J, Gu L, et al. Extent of surgery for papillary thyroid cancer is not associated with survival: an analysis of 61,775 patients. Ann Surg. 2014;260: 601-605.

32. Haigh PI, Urbach DR, Rotstein LE. Extent of thyroidectomy is not a major determinant of survival in low- or high-risk papillary thyroid cancer. Ann Surg Oncol. 2005;12:81-89.

33. Barney BM, Hitchcock YJ, Sharma P, Shrieve DC, Tward JD. Overall and causespecific survival for patients undergoing lobectomy, near-total, or total thyroidectomy for differentiated thyroid cancer. Head Neck. 2011;33:645-649.

34. Mendelsohn AH, Elashoff DA, Abemayor E, St John MA. Surgery for papillary thyroid carcinoma: is lobectomy enough? Arch Otolaryngol Head Neck Surg. 2010;136:1055-1061.

35. Matsuzu K, Sugino K, Masudo K, et al. Thyroid lobectomy for papillary thyroid cancer: long-term follow-up study of 1,088 cases. World J Surg. 2014;38:68-79. 